\title{
Summary of papers presented at the meeting of the Society for Social Medicine in Aberdeen, September 1982
}

\section{Increasing cost of NHS hopitals}

A M G R A Y, Health Economics Research Unit, Aberdeen Much concern has been expressed about the cost of hospital care, but the last full study of hospital expenditure was conducted by the Guillebaud committee in 1956. Inflation has been higher in the NHS than in the economy as a whole; health expenditure deflated by the retail price index shows a "real" growth of $160 \%$ between 1950 and 1977 , but using a health service price index the real increase is $108 \%$.

The hours worked by most types of staff have fallen: a whole time equivalent (WTE) nurse in 1982 worked 22⿺ fewer hours than in 1950. Scottish data suggest that about one third of the WTE increase in nursing numbers can be explained by falling hours, and this suggests that present estimates of increases in health service inputs must be inaccurate and misleading. As Guillebaud found in 1956, so in 1982 it seems clear that the standard NHS accounts are inadequate and need to be closely scrutinised and recalculated.

Benefits and costs of a ban on the sale of non-pasteurised milk in Scotland

D $\mathrm{R}$ COHEN, Health Economics Research Unit, Aberdeen

The benefits of a ban on the sale of non-pasteurised milk in Scotland are viewed as reductions in the incidence of milk borne salmonellosis based on the costing of a recent major outbreak in Grampian Region. The monetary costs of this outbreak are estimated at $£ 83230$ with an additional cost of between $£ 150135$ and $£ 3131090$. Based on annual rates of incidence, the annual monetary benefits of the ban will be $£ 31943$ and the total benefits will be between $£ 89607$ and f1 233655 a year. It is suggested that the costs of the ban are unlikely to exceed the benefits under all but the most severe assumptions.

Street-level bureaucrats: the real health policy makers? D J HUNTER, Unit for the Study of the Elderly, Aberdeen

The NHS is more decentralised in its operation than is often appreciated, and the centre's hold over the direction of health policy is weak. Data derived from studies of resource allocation and priority setting processes, including a study of the allocation of development funds in Scotland, show this. The shift towards services for the "Cinderella" sectors-that is, national priority groups-has been faltering, uneven, and occasionally non-existent. In general, national policy guidelines have not been successful in determining local action. If a consensus exists at all levels then local actions are likely to accord with the centre's preferences. If not the ability of the periphery to thwart, distort, and delay should be neither overlooked nor underestimated.

Disadvantaged families: continuity between generations in health attitudes

M B AXTER, Institute of Medical Sociology, Aberdeen This study examined in a sample of 58 three-generation families in poor socioeconomic circumstances the relationship between attitudes to health and behaviour in the behaviour in the health care of children. Continuities or discontinuities between generations were studied by longitudinal survey of health events, by tape recorded interviews, and by the use of records.

There was little evidence of direct mother to daughter transmission of attitudes or influence on behaviour. Though there was some perpetuation of familiar or subcultural ideas about health, there were also many changes between the generations. Attitudes to preventive care remained similar where the environment of the family had changed least. The persistence of health deficit in children of poor families may be attributed rather to continuity in a disadvantaged environment and the inevitable effect on the behaviour of young mothers of the constraints of that environment.

\section{Normal aging: a longitudinal study}

G FOR D, Institute of Medical Sociology, Aberdeen

It is increasingly clear that much of the variance in life satisfaction is not explicable by traditional structural variables, and the Aberdeen longitudinal study of normal aging shifts the emphasis from states of psychological wellbeing to processes of adjustment to, and coping with, stressful events. Nineteen resource variables covering six life domains were subjected to cluster analysis, and 10 resource profiles were derived. Each cluster was shown to have implications for the capacity to resist stressful events.

\section{Examining trends in cancer rates over time}

M J GARDNER and C OSMOND, MRC Environmental Epidemiology Unit, Southampton

There is current controversy over whether or not death rates from cancer are increasing as new chemicals and other substances recently introduced into the environment may be carcinogenic. Time trends for cancer suggest an increasing rate using both crude death rates and age standardisation death rates. Crude death rates can be misleading from an epidemiological viewpoint, but age standardised death rates may also be inappropriate. For instance, the action of carcinogens that affect some generations more than others can produce what appear to 
be secular trends even with age standardisation. A statistical model that overcomes this, separating the effects of age, period of death, and period of birth on disease trends over time, was compared with simple age standardisation. The application of the model showed some major cohort effects, both increases and decreases. No evidence supported the view that there is an increasing period effect in cancer mortality.

Cause of death among a national sample of registered patients with cancer

D A LEON, A ADELSTEIN, P GOLDBLATT, and A J FOX, The City University, London School of Hygiene and Tropical Medical, and Office of Population Censuses and Surveys

During 1971-5 some 8000 people in the OPCS Longitudinal Study were registered with cancer. The longitudinal study is a $10 \%$ sample of the 1971 England and Wales Census to which various subsequent vital registration events are linked. Of the 4190 men and 4005 women, 3047 (73\%) and 2367 (59\%) respectively died in the follow up period $1971-6$. About $10 \%$ of these deaths were from causes other than cancer. Standardised mortality ratios for causes other than cancer were extremely high shortly after registration. In the first fortnight the SMR was around 1000 , but by six months it had fallen to about 100 . A substantial part of this early excess could be attributed to mortality from pneumonia. The findings may be partly explained in terms of problems in perception of the "underlying cause" of death for people who die soon after registration as well as by adverse effects of treatment.

Long term survival, causes of late death, and incidence of second primary tumours after childhood cancer

M M HAWKINS and G J DRAPER, Childhood Cancer Research Group, Oxford

A preliminary analysis has been carried out of the rates and causes of late deaths and the occurrence of second primary tumours among over 5000 children diagnosed with cancer before 1971 and surviving at least three years. Those with embryonal tumours, particularly retinoblastoma and Wilms' tumour, showed the highest survival rate; of those surviving three years, over $90 \%$ were alive 20 years later. The corresponding figure for neuroblastoma was about $80 \%$. About half of those suffering from Hodgkin's disease, medulloblastoma, Ewing's tumour, or rhabdomyosarcoma survive a similar period. The estimated probability of a second primary tumour did not exceed $4 \%$ during the 20 years after three year survival, with one exception, bilateral retinoblastoma, where the figure was about $10 \%$.

A randomised controlled trial of the value of stilboestrol treatment in pregnancy: long term follow up of mothers and their offspring

M P VESSEY, D V I FAIRWEATHER, and B NORMAN-SMITH, Department of Community Medicine and General Practice, Oxford, and Department of Obstetrics and Gynaecology, University College, London Stilboestrol was used much less during pregnancy in the United Kingdom than in the United States, but attention was drawn some years ago to a randomised controlled trial of the treatment among over 1000 primigravidas conducted at a London teaching hospital in the early 1950s. Details have been obtained of deaths, cancer registration, and certain other disorders occurring among them. The data currently available are incomplete, but there is little evidence so far of any serious effect of the use of stilboestrol in pregnancy on morbidity or mortality in either the mothers or their offspring, save for a possible increase in psychiatric problems in the offspring.

An evaluation of bladder cancer mortality data by use of cancer registry data

J M DAVIES and R G SKEET, Institute of Cancer Research, Sutton, Surrey

Death certificate data for all 438 men resident in the area covered by the South Thames Cancer Registry and certified as dying of bladder cancer during 1978 were compared with corresponding registration data. Of the decedents, $3.5 \%$ had cancer of a different site (usually prostate) roughly balanced by "false negative" cases with death wrongly attributed to cancer of a different site than the bladder. About $65 \%$ of the decedents had their cancers first diagnosed during 1977-8, 22\% during 1974-6, and 13\% earlier; $5 \%$ of the decedents had changed their "district of usual residence" between registration and death. At ages under 65 the total of 1978 deaths from bladder cancer was only $32 \%$ of the total of 1977 registrations of bladder cancer; at ages over 65 it reached $53 \%$.

Case control study of breast cancer in north east Brazil: preliminary results

A KALACHE, P SMITH, and R DOLL, Cancer Epidemiology and Clinical Trials Unit, Oxford

The rule is that the poorer the country, the lower the risk of breast cancer, but north east Brazil is a remarkable exception: an underdeveloped area where incidence rates are nearly as high as in Britain. Clues to disease aetiology may often be gleaned by studying exceptional situations, and in April 1980 a case control study was set up in Recife and Fortaleza where 650 cases were interviewed together with their hospital and neighbourhood controls (one of each per case). Preliminary analysis seems to indicate that aspects related to women's reproductive histories and to diet are important risk factors for breast cancer in north east Brazil.

Statistical analysis of a case control and cohort study of cigarette smoking and lung cancer in the west of Scotland D J HOLE, C R GILLIS, V M HAWTHORNE, and $P$ B O Y LE, Cancer Surveillance Unit, Glasgow, and School of Public Health, University of Michigan

A hospital based case control study of 656 men with lung cancer, 1312 age and sex matched controls, and a cohort study of 7390 men aged 45-64 drawn from the general population of urban west of Scotland were examined to ascertain the extent to which cigarette smoking was responsible for the high rate of lung cancer. The dose response relationship was curvilinear in both studies with little increase in risk for smokers of more than 25 cigarettes 
a day. An exposure index containing number of years of smoking and tar yield provided a best goodness of fit to the data using a multiple logistic regression approach. Lower tar, however, had little effect in reducing risk for those smoking more than 15 cigarettes a day. Comparison with other major cohort studies suggested that the west of Scotland smoker was at twice the risk of lung cancer compared with his equal smoking counterpart.

\section{Aetiological factors in lymphoid malignancies: a case control study}

S M BERNARD, R A CARTWRIGHT, I D G RICHARDS, and C C BIRD, Department of Community Medicine and General Practice, Department of Pathology, and Yorkshire Regional Cancer Organisation, Leeds

A pilot case control study of adult lymphomas and lymphocytic leukaemias was conducted in selected areas of the Yorkshire Health Region from 1979 to 1981 . For each case a control matched for age and sex was selected from the hospital inpatient population and interviewed. Analyses of the first 285 cases and their matched controls indicate an excess of Jews relative risks $(R R)=4 \cdot 49$, a previous history of eczema/dermatitis $R R=3.78$ and skin cancer $R R=7 \cdot 18$, benzodiazepine use for non-Hodgkin's lymphoma only, $R R=2 \cdot 85$, and solvent exposure $R R=1 \cdot 81$. The study has recently been expanded into a major study covering the whole of the Yorkshire Health Region and including all lymphomas and leukaemias.

\section{Audit of the quality of medical records}

F G R FOWKES, S M PAGE, and D PHILLI PS - M ILES, Department of Epidemiology and Community Medicine, Cardiff, and West Glamorgan Health Authority

A stratified random sample of 285 patient discharges from the general medical units in two hospitals was selected from the Hospital Activity Analysis listing of all discharges in one year with a principle diagnosis of myocardial infarction, bronchitis, and cerebrovascular accident. Data on the accuracy of the filing within the records, the completion of standard forms and the recording of events in the history and physical examination were obtained from the medical records. The admission and progress notes were filed correctly in over $90 \%$ of records in contrast to the filing of discharge summaries and correspondence, which was correct in $62 \%$ of records. An $x$ ray report was present in the case notes for only $24 \%$ of the occasions that patients had undergone radiography. The recording of events in the history and physical examination were below the minimal standard indicated by the consultants before the audit. These and other results suggest that an improvement in the quality of medical records is desirable.

\section{Screening or case finding for the detection of hypertension in general practice}

A J SILMAN, C M LOCKE, F M MURPHY, and G J W A LKER, Department of Epidemiology, London Hospital Medical College, London.

Two methods, have been advocated for detecting hypertension in general practice: "case finding" where the general practitioner uses any patient contact to measure blood pressure and "screening clinics," specially organised. A randomised trial was conducted comparing the two methods among 20 general practitioners and their 8000 patients aged 50 to 64 . Results from the first 18 months show that case finding covered $50.4 \%$ of the population with a pronounced fall, unexplained by attendance rates, over time. In the screening clinics $58 \cdot 3 \%$ of the patients attended. In both groups attendance was higher among women and increased with age. In the screening group $21.4 \%$ had a diastolic blood pressure greater than $95 \mathrm{~mm} \mathrm{Hg}$ compared with $18.2 \%$ in the case finding group. In the case finding groups a higher proportion had been diagnosed as having hypertension than in the screening group. Case finding in general practice is a more cost effective method of detecting previously undiagnosed hypertension than screening clinics, but screening clinics have a greater yield, are more likely to detect lapsed hypertensive patients, and are not subject to the same problem of declining interest.

Use of inner London accident and emergency departments: the relationship with the availability of general practitioners J S CHAMBERS, R D T FARMER, and D R JONES, Department of Community Medicine, Westminster Medical School, London

In a cross sectional study of 3824 new patients attending six accident and emergency departments in inner and outer London during one week young men and those living in single person households accounted for high proportions of the attenders. A much higher proportion of local patients in inner and outer London had no general practitioner available to them in the area where they were currently living. In inner London accident and emergency departments proportionately more patients had conditions appropriate for general practitioner treatment, minor medical complaints, and had delayed treatment for more than 24 hours. Non-registered patients tended to present with more minor complaints than registered patients. Differences between inner and outer London accident and emergency departments could not be explained in terms of demographic factors and general practitioner registration.

Giving up smoking: the attribution and consequences of success

K D JAMROZIK, M P VESSEY, G H FOWLER, and N J W A LD, Department of Community Medicine and General Practice, and Cancer Epidemiology and Clinical Trials Unit, Oxford

A sample of 100 patients who stated that they were not smoking at the time of a one year postal follow up were interviewed at home. One third of the patients admitted to having started smoking again, most commonly after some upset in their personal life. Of those who did not relapse, only $30 \%$ thought that their doctor had been helpful, over half reported an improvement in their general health, and a similar proportion did not miss smoking. A few reported transient stress with spouses, and of gains in weight, but there was no evidence of increased use of alcohol or anxiolytics. These data may be of use to health professionals in counselling individual smokers who fear that the costs of stopping may outweigh the benefits. 
Obtaining an abortion: pathways of referral for Camden women

L CLARKE, C FARRELL, and B BEAUMONT, British Pregnancy Advisory Service, Polytechnic of North London, and Islington District Health Authority, London The Camden Abortion Study investigated the reasons why a decreasing proportion of women residents had been obtaining NHS abortions. One third (31\%) of the women went straight to private pregnancy advisory bureau doctors and there was little referral from these doctors to the NHS (only $1 \%$ of total sample); over two thirds (69\%) of the women first consulted an NHS doctor (usually their general practitioner) and over one quarter of them $(28 \%, 19 \%$ of total sample) had their operation privately. Preference for NHS treatment centred around the cost of a private abortion, which was shown to be considerable.

One fifth of the women did not consult their general practitioner, mainly for reasons of privacy or expectation that their general practitioner would be unsympathetic. Also one third of those who had private operations thought that it was fairly or very difficult to get an NHS abortion. Nearly half of the women named the speed of referral as the main reason why they had a private abortion. Over half (53\%) the women who visited a pregnancy advisory bureau had their operation within within one week of their first visit. One source of delay for women having NHS operations was caused by pregnancy testing, which was carried out at hospitals, but repeated visits to general practitioners and waiting times for hospital appointments also played a part.

Maternal factors and birth weight: a comparative analysis T J PETERS, J G FRYER, C J LA WRENCE, J GOLDING, and N R BUTLER, Department of Mathematical Statistics and Operational Research, Exeter, and Department of Child Health, Bristol

The two national birth cohort studies consisting of surveys among children born in a particular week (3 - 9 March 1958; 5-11 April 1970) have provided a comparison of the associations of birth weight with social class, maternal age and height, parity, smoking, and hypertension during pregnancy over the intervening period. The birthweight distributions across the categories of the maternal variables individually were considered first, followed by an analysis of covariance on each data set. Modifications to the linear model such as regrouping the variables, interactions, and the extraction of subpopulations were then investigated. Fitted constants were obtained to indicate the groups of mothers at risk as regards birth weight. Considerable changes in the maternal population and obstetric management were observed between the two surveys; however, the dominant factors in relation to birth weight have remained the same-maternal height, smoking history, parity, and hypertension.

Relationship of neonatal BCG vaccination to the incidence of tuberculosis in childhood

I LECK, H M CURTIS, and F N BAMFORD, Departments of Community Medicine and Child Health, Manchester

Neonatal BCG vaccination has long been offered routinely in one hospital (hospital A) where nearly two fifths of infants of Manchester residents are born. This is not the practice elsewhere in the city. Among children born to Manchester residents in 1965-80, 65 were ascertained as having presented with tuberculosis in 1975-80 while still residing in the city, of whom 10 (four vaccinated and six unvaccinated) had been born in hospital $A$. The results for unvaccinated children born at hospital $A$ and for the children born elsewhere were closely similar to each other, and more than four times as high as the figure for children born at hospital $\mathbf{A}$ and vaccinated. Children with Asian surnames and others both shared in the reduced risk associated with vaccination.

Prediction of infant feeding: a contribution to health education

M R HALLY, J BOND, B A GREGSON, and I T RUSSELL, Health Care Research Unit, Newcastle

Factors influencing mothers in their choice and use of an infant feeding method were studied in a random sample of 500 mothers. From the 34th week of pregnancy until six months after delivery, data were collected by home interviews and postal questionnaires, and from hospital case notes. At the time of their first antenatal hospital visit, three quarters of the mothers had already decided on their intended method; nearly all adhered to this choice. Factors which together predict whether or not each mother will breast feed were identified by multivariate statistical analysis; $93 \%$ of mothers studied could have been allocated correctly. A scoring system based on these factors would enable health professionals to identify in advance the "borderline" mothers who might well breast feed successfully.

Changes in dental caries experienced by English 5 year old children: 1947-80

D J A CKSON, Department of Community Medicine and General Practice, Leeds

From 89 epidemiological studies of dental caries in English 5 year old children living in non-fluoridated areas it has been possible to study trends between 1947 and 1980 . Between 1947 and about 1960 experience of caries rose slightly but since about 1960 there has been a gradual fall with the steepest decline occurring between 1977 and 1980. Whereas the rise in caries between 1947 and 1960 was associated with the postwar period of increased consumption of sugar and sweets, the later fall cannot be similarly correlated with a corresponding fall in the consumption of these commodities. In non-fluoridated areas the major source of the fluoride ion available to teeth is in toothpastes. Fluoridated toothpastes were introduced into the British market in the early ' 70 s, and gradually the proportion of $\mathrm{F}$ toothpastes to all sold toothpastes rose to $98 \%$ by 1980 . Whereas the fall beginning in 1960 or thereabouts occurred too early to be associated with the use of fluoride toothpastes, the accelerated fall beginning about 1977 is associated. These changes have probably been caused by a multiplicity of factors, none of which can be identified with certainty. The operating factors appear to have induced a parallel decline in fluoridated and non-fluoride communities still obtains. 
Behavioural development and educational attainment in a cohort of young children followed for four and a half years I A BAKER, S J HUGHES, E C STREET, and P M SWEETNAM, Bristol and Western Health Authority, MRC Epidemiology Unit, Cardiff, and South Glamorgan Health Authority

Fourteen of 480 children in a birth cohort were found by using the Richman behaviour screening questionnaire to have behaviour problems at the age of 5 years. Twelve of these 14 children (cases) were seen again at the age of $8 \frac{1}{2}-9$ years, together with 24 control children matched for age, sex, and social class. Using the Rutter parental questionnaire, seven of the 12 cases had behaviour problems at $8 \frac{1}{2}-9$ years compared with four out of 24 control children $(\mathrm{p}<0.05)$. By the Bristol social adjustment guide completed by teachers, 11 of the 12 case children had abnormal behaviour reactions compared with 10 of the 24 control children $(p<0.05)$. The Pidgeon non-verbal test BD showed no difference in non-verbal IQ between the case and control children. The Neale analysis of reading ability indicated that the case children were 5 months behind the control children for age of reading accuracy and comprehension. These differences were not statistically significant, nor thought to be important educationally.

Positive discrimination and the role of health visitors in deprived inner city areas

S GRIFFITHS, S BROWN, W BERESFORD, and M SARKHOV, London School of Hygiene and Tropical Medicine, London

A questionnaire and a diary for a working week allowed description of health visitors' perceptions of their role and priorities to be compared with their practice in relation to the social characteristics of their clients. Health visitors perceived themselves ideally to be concerned with prevention and health education but they felt that they were involved with crisis intervention and referral at the expense of these functions. When the recorded workload was divided into priority and non-priority cases, aggregation of health visiting function for the priority group showed that crisis intervention occurred in $33 \%$ of cases compared with $3 \%$ non-priority cases. Aggregation of the social characteristics of this priority group showed that among these cases there was a significant overrepresentation of mothers under age 20, unsupported mothers, and unemployed fathers. Families were more likely to be receiving social security, and social workers were concerned in $36 \%$ of the cases.

Effect of health visitors working with the elderly in general practice: a randomised controlled trial

N J VETTER, D A JONES, and C R VICTOR, Research Team for the Care of the Elderly, Cardiff

A total of 1288 people aged 70 and over and living at home in two general practices in south Wales were interviewed to assess their physical, mental, and social status and their use of services. A health visitor was employed in each practice specifically to care for a random sample of these people over a two year period: 572 people were so visited, 571 acted as controls. At the end of the two years, all of the study group were reinterviewed using a schedule similar to the first. The health visitor in the urban practice had a pronounced effect in providing extra services for those individuals allocated to her when compared with the controls. The rural health visitor had no such effect. The elderly who were on the health visitor's list in the urban practice had a lower mortality than those who were not. There was no difference in mortality in the rural practice. No significant differences in physical, mental, or social status were detected in either intervention or control group.

A population based survey of the elderly: family networks and support

A ODELL, S CLARKE, and M CLARKE, Department of Community Health, Leicester

A survey was undertaken in Melton Mowbray, Leicestershire, of all those aged over 75 registered with general practitioners to describe their characteristics and to determine the help and services needed to maintain them at home. Preliminary results showed that for a sizeable minority of elderly people family members are either not available or not in contact: $63 \%$ had no spouse, $23 \%$ had no children, and $17 \%$ had neither. Of the $46 \%$ interviewed who lived alone, $47 \%$ had had fewer than four contacts with family members in the previous week. Contact with family not living in the same household decreased with age and increased with lower social class.

Services for disabled people: what criteria should we use to assess disability?

S SOMER VILLE, R SILVER, and D PATRICK, Department of Community Medicine, St Thomas's Hospital, London

The measure of disability used in the UK National Sample Survey (NS) of the disabled in 1969, based solely on self care activities, was compared with the functional limitations profile, a much broader measure of disability on the same 748 disabled people in Lambeth. Altogether 209 were classified as severely/very severely disabled on the functional limitations profile compared with only 40 on the NS measure. Of the 209, 126 had either no disability or only minor disability on the NS instrument. In terms of health and social service use, restriction in ambulation mobility, and household management these 126 were quite similar to the 109 who had appreciable to very severe disability on the NS measure. The NS measure fails to identify many disabled people who might need services but is the DHSS recommended guide for local authority surveys to estimate need. The DHSS should contine the evaluation and development of measures of disability.

\section{Social support and the course of disability}

M MORGAN, D PATRICK, and J CHARLTON, Department of Community Medicine, St Thomas's Hospital, London

The relationship between social support and the course of disability was examined in a sample of 585 people aged 45-75 living at home and having some functional limitation or activity restriction. A low level of social contact was significantly associated with deterioration in psychosocial disability over a two year period and in both psychosocial and emotional disability over a 12 month period, with the 
effect of level of support being greatest in the presence of adverse life events. These differences held even when age, sex, and initial level of disability were controlled. The same pattern existed for physical disability, although more weakly. There was, however, no significant relationship between level of emotional support and the course of disability.

Illness, drugs, and disability among those aged 16-75 living at home

H PEACH and J CHARLTON, Department of Community Medicine, St Thomas's Hospital, London

A prevalidated medical questionnaire was incorporated into an interview with a sample of disabled adults from London borough. Stroke and depression were associated with physical disability and sciatica and hypertension with social disability. Most symptoms associated with disability had been reported to doctors and were being treated. Some symptoms associated with disability might be the side effects of drugs. Prevention of stroke would reduce disability in the community but otherwise the possibilities for primary prevention are limited. Since many symptoms associated with disability have been reported to doctors and are being treated there is little likelihood of reducing disability through increasing general practitioners' awareness and treatment of impairments. There might be scope for reducing iatrogenic disability.

A prospective study of vegetarians and meat eaters M THOROGOOD and J I MANN, Department of Community Medicine and General Practice, Oxford In 1980 a prospective study was started of 6000 vegetarians, who were asked to name non-vegetarian, age matched friends to form a control cohort. Detailed questionnaires including dietary data were completed, and the NHS records of all participants were flagged. Baseline data on 2000 cases and 1000 controls have been analysed. Social class is similar in the two cohorts, but differs appreciably from the general population, with under $10 \%$ being manual workers. Current smoking was uncommon (13\% vegetarians, $22 \%$ controls). Intake of dietary fibre was greater among vegetarians (around $31 \mathrm{~g}$ a day compared with $22 \mathrm{~g}$ ). It is hoped that this study will provide information about the mortality experience of vegetarians and will enable some examination of which environmental factors may be important.

Vegetarianism, dietary fibre, and mortality $M$ L BURR and $P$ M SWEETNAM, MRC Epidemiology Unit, Cardiff

The effects of vegetarianism and dietary fibre on mortality were examined by means of a cohort study. Customers of health food shops and readers of "health" journals were recruited by means of a short questionnaire. The NHS records of 10943 subjects were flagged, and their mortality was analysed after the first seven years. Intake of fibre was not significantly associated with mortality, although those who ate wholemeal bread daily had fewer deaths from cerebrovascular disease. Vegetarians had a significantly lower mortality from ischaemic heart disease than non-vegetarians, the difference occurred mainly in the men and was not due to smoking. It confirms other evidence of less heart disease in vegetarians, which may be attributable to their abstinence from meat, their high intake of vegetables, or non-specific factors in their general way of life.

Dietary fibre and acute appendicitis

M NELSON, and D J P BARKER, MRC Environmental Epidemiology Unit, Southampton

A study of acute appendicitis in nine British towns showed that the three northern towns had the highest incidence. Food sales data for each town showed that these three northern towns had relatively greater consumption of potatoes in relation to other vegetables, and relatively greater consumption of brown and wholewheat bread in relation to white bread. These findings suggested higher cereal fibre intake and lower vegetable fibre intake in communities with higher rates of appendicitis. To explore this further the diets of 30 Southampton schoolchildren aged 13-15 who had histologically confirmed acute appendicitis in 1981-2 were compared with the diets of 60 matched controls. Diet was measured by a seven day weighed inventory. Intakes of carbohydrate, water from all foods, and dietary fibre from cereals and potatoes were slightly higher in controls than cases, but the differences fall short of statistical significance. The influence of dietary fibre on incidence of disease between communities in Britain therefore appears to differ from its influence within communities.

How long should a dietary survey be?

J A HEADY and J W MARR, Department of Clinical Epidemiology and General Practice, Royal Free Hospital, London

The use of dietary data in epidemiological studies depends on the reliability with which individuals' diets can be classified. Intakes vary between individuals and within individuals from day to day. The key factor in identifying the men in the top and bottom thirds of the distribution of any nutrient is the ratio of the between individual to the within individual variation. From this ratio the number of days necessary to record intakes to achieve a given reliability may be calculated. Using seven day weighed surveys from civil servants and London bus drivers to achieve $80 \%$ correct classification in the extreme thirds of the distribution of energy intake, for example, it required a seven day survey for civil servants but only four days for the drivers. A one day survey correctly classified only $60 \%$ of the civil servants and $67 \%$ of the drivers, with $9 \%$ and $6 \%$ being grossly misclassified for energy intake.

A survey of dietary nitrate in users of water from wells C CHILVERS, H INSKIP, P FRASER, C CA Y GILL, and B BART HOLOMEW, Institute of Cancer Research, Sutton, London School of Hygiene and Tropical Medicine, Public Health Laboratory Services, Colindale, and Bacterial Metabolism Research Laboratory, Porton Down

A survey among 476 users of water from wells in Norfolk 
enabled the relative contributions of food and water to total nitrate intake to be ascertained over a wide range of water borne nitrate levels. Samples of drinking water, 24 hour urine specimens, and 48 hour diet diaries were obtained on two occasions from each participant. A clear relationship emerged between intake of nitrate from water and excretion of urinary nitrate after allowing for nitrate ingested in food, principally from vegetables and cured meat products. The proportion of nitrate ingested from drinking water exceeded $50 \%$ of the daily intake only where the concentration of nitrate was greater than the EEC recommended maximum of $50 \mathrm{mg} / \mathrm{l}$. Since there is some evidence that high ingestion of nitrate may increase the risk of gastric cancer by acting as a precursor in the formation in vivo of carcinogenic $\mathrm{N}$-nitroso compounds, these results suggest that current standards of drinking water should be maintained.

\section{Alcohol intake and biochemical markers} D DAVIS, S J POCOCK, M WALKER, and A G SHAPER, Department of Clinical Epidemiology and General Practice, Royal Free Hospital School of Medicine, London

In a clinical survey of 7735 men aged 40-59 alcohol intake was estimated using an administered questionnaire. Blood samples were taken and 25 biochemical and haematological variables determined for each man. Many variables were associated with alcohol consumption, gamma-glutamyl-transpeptidase and lead being most strongly associated. Mean corpuscular haemoglobin mean corpuscular volume, high density lipoprotein cholesterol, urate, and aspartate transaminase also showed substantial associations with alcohol intake. When heavy drinkers were compared with occasional drinkers, CGTP was the best single predictor of heavy drinking. Better identification of heavy drinking was obtained by using a combination of measurements. Using discriminant analysis technique, the best five variable discriminant was based on GGTP, HDL-C, urate, $\mathrm{MCH}$, and lead. In epidemiological studies concerning alcohol the use of biochemical/haematological indices should complement but not replace the history of alcohol consumption.

Positive discrimination in child health: problems of implementation and evaluation

R J MADELEY, Department of Community Health, Nottingham

In Nottingham North and South Health Districts an attempt was made to predict which babies were more likely to die in the postneonatal period and they received extra attention from health visitors and general practitioners. At the time of introduction of the scheme, a control group was considered to be unethical, so that it was introduced for all "high risk" infants. This has naturally led to some problems of evaluation. Confirmed postneonatal mortality rates for 1979 and 1980 , and provisional ones for 1981 , have shown a considerable fall, greater than the national average, so that the rate in the City of Nottingham is now no higher than the national average.
First steps to monitoring regional strategic planning F ALBERTONI and S M HOWIE, London School of Hygiene and Tropical Medicine, London

An attempt is made to monitor the implementation of the 10 year (1979-88) strategic plan of an English health authority. There is some evidence of redistribution of resources towards the region's priority groups (elderly, mentally ill, mentally handicapped, and children) at the expense of acute and general hospital services, but there is little evidence of shift towards geographical equity or to community services. Increased efficiency in terms of hospital care is found, but no shift towards community care in the region as a whole is seen, although some districts do show progress. Such indicators could be integrated into the NHS strategic planning system. This exercise makes a first step towards planning for need, which should concern those provided for as well as those providing services.

Evaluation of computed tomography: a review of research methods

I T RUSSELL, Health Care Research Unit, Newcastle Diagnostic technology can be evaluated at four different levels-technical capability, diagnostic accuracy, therapeutic impact, and patient outcome. Although outcome is arguably of most concern to patients, it is the most difficult to evaluate. Few publications on computed tomography report on outcome. Even more disappointing is the inadequacy of the research methods used to evaluate CT. The results of only one randomised trial have been published, and none of the many before and after studies has been properly controlled. Despite these methodological shortcomings, the diagnostic superiority of $\mathrm{CI}$ is so striking that it may be regarded as established. In contrast, the evidence that CT is effective in improving patient outcome is still weak. To evaluate properly nuclear magnetic resonance, the latest innovation in diagnostic technology, research methods sounder than those used to evaluate CT will be needed.

Mortality in British firemen: a cohort study with a "healthy worker" standard

S DONNAN, Department of Community Medicine, Chinese University of Hong Kong, Hong Kong

A cohort of 5574 firemen was assembled from five brigades in England to investigate suspected increased risks of heart and lung disease. Between 1965 and 1979, 64000 man years at risk were studied, with death as the outcome. Because the "healthy worker effect" was expected, standard rates were obtained from the OPCS Longitudinal Study for 1971-5 based on employed or retired men; for the regions, the rates were based on all men. A total of 170 deaths were observed, 82 with ischaemic heart disease as underlying cause. The SMRs based on LS employed/retired rates ranged from 0.57 to 0.99 for the five brigades. Regional adjustment of the LS employed/retired rates brought all the lower SMRs closer to $\mathbf{1 \cdot 0}$. For ischaemic heart disease, in most brigades some or all of the age specific mortality ratios were over $1 \cdot 0$, significantly so for one northern brigade. Respiratory and other diseases showed no increased SMRs. Current firemen were shown to smoke no more cigarettes than average, but smoking information 
was not available for ex-firemen. This study is consistent with previous reports of slight increased risk of ischaemic heart disease in firemen. Continuation of physical fitness training for firemen is warranted.

Effect of death certification and coding practices on observed patterns of respiratory disease mortality in the EEC $M$ KELSON, M FAREBROTHER, and R F HELLER, Department of Community Medicine, St Thomas's Hospital, London

The study was designed to see if variations in death certification and coding practices in eight EEC countries could account for observed differences in respiratory disease mortality. In each country a representative sample of doctors was asked to complete a death certificate for each of 10 case histories. Completed certificates were coded at the national offices and the assigned codes grouped into broad diagnostic categories. The certificates were then coded at a World Health Organisation reference centre to compare national codes against a standard coding procedure. Systematic between country differences in the diagnostic categories used explained some of the between country differences in respiratory disease mortality. These were due partly to ways in which doctors certified deaths and partly to national coding variations. International comparisons based on mortality statistics should be treated with considerable caution until greater standardisation of certification and coding practices in the EEC is achieved. 